\title{
Outflowing ionospheric oxygen-ion motion in a reconfigurating magnetosphere
}

\author{
E. B. Wodnicka, M. Banaszkiewicz \\ Space Research Centre, Bartycka 18a, 00-716 Warsaw, Poland \\ Received: 3 July 1995/Revised: 18 July 1996/Accepted: 22 July 1996
}

\begin{abstract}
During substorms, large-scale changes of the topology of the Earth's magnetosphere following the variation of the characteristics of the interplanetary medium are accompanied by the induction of the electric field. In this study a model of a time-dependent magnetosphere is constructed and the large-scale features of the induced electric field are described. Local-time sectors with upward or downward field-aligned component and with intense perpendicular component of the electric field are distinguished. The electric-field structure implies the existence of outflow regions particularly effective in ion energization. With the vector potential adopted in the study, the region from which the most energized ions originate is defined by the local-time sector near 2100 MLT and latitude zone near $71^{\circ}$ MLAT. The motion of ionospheric oxygen ions of energy $0.3-3 \mathrm{keV}$ is investigated during a 5-min reconfiguration event when the tail-like magnetospheric field relaxes to the dipole-like field. As the characteristics of plasma in the regions near the equatorial plane affect the substorm evolution, the energy, pitch angle, and the magnetic moment of ions in these regions are analyzed. These quantities depend on the initial energy and pitch angle of the ion and on the magnetic and electric field it encounters on its way. With the vector potential adopted, the energy attained in the equatorial regions can reach hundreds of $\mathrm{keV}$. Three regimes of magnetic-moment changes are identified: adiabatic, oscillating, and monotonous, depending on the ion initial energy and pitch angle and on the magnetic- and electric-field spatial and temporal scales. The implications for the global substorm dynamics are discussed.
\end{abstract}

\section{Introduction}

The idea of the high-latitude ionosphere coupling with regions of the magnetosphere is supported by data of

Correspondence to: E. B. Wodnicka increasing resolution from multipoint observations and by modeling.

In the statistical study of Yau et al. (1984) the DE1 ionospheric upflowing ion (UFI) data in the energy range $\varepsilon=0.01-17 \mathrm{keV}$ were binned according to altitude, local time, latitude, and magnetic activity (two levels: $K p<3$ and $K p>4$ ). The findings relevant to $\mathrm{O}^{+}$ions will be quoted. Intense fluxes of $\mathrm{O}^{+}$UFIs occurred in an ovalshaped region, for $\varepsilon>1 \mathrm{keV}$ closely associated with the auroral oval. Fluxes were most intense and most variable (with flux density in the interval $2.3-9.6 \times 10^{24}$ $\mathrm{cm}^{-2} \mathrm{~s}^{-1} \mathrm{sr}^{-1}$ ) in the energy range $0.01-1 \mathrm{keV}$ and for $160^{\circ}-180^{\circ}$ pitch-angle ions, and the low-energy UFIs occurred uniformly in local time. Marked magnetic activity dependence for $\mathrm{O}^{+}$UFIs was found.

A longer period of data (September 81-May 84) analyzed in the subsequent study of Yau et al. (1985) allowed to estimate long-term variation of UFI fluxes. The $\mathrm{O}^{+}$outflow rate correlated with the declining solar radioflux and, at active times, ranged from $1.5 \times 10^{26}$ ions s $^{-1}$ in the solar maximum period to a value twice as small in the period of reduced solar activity. An exponential increase of the $\mathrm{O}^{+}$-ion outflow rate with the $K p$ index was established: $8 \times 10^{24}$ ions s $^{-1}$ at $K p=0$ and $2.5 \times 10^{26}$ ions s $^{-1}$ at $K p \gg 6$. The distribution of UFIs peaks near $78^{\circ}$ ILAT in the noon sector. In the nightside, for $0.01-1-\mathrm{keV}$ ions, it peaks at lower latitudes above $62^{\circ}$ ILAT.

Chappell et al. (1987) provided arguments that the observed densities in the plasmasphere, plasma trough, plasma sheet, and in the lobes can be attained with no contribution from the solar-wind plasma but exclusively from the ionosphere. Three sources were considered: the auroral zone $\left(10-100 \mathrm{keV}, 3.2-7.7 \times 10^{25}\right.$ ions s $\left.^{-1}\right)$, cleft $\left(2-20 \mathrm{eV}, 1.9-4.8 \times 10^{25}\right.$ ions s$\left.^{-1}\right)$, and the polar cap $\left(10-100 \mathrm{eV}, 1.5-2.5 \times 10^{25}\right.$ ions s $^{-1}$, at active times).

Measurements of the substorm UFI fluxes on-board DE1 and DE2 located on the common magnetic field lines in the auroral premidnight sector were interpreted by Reiff et al. (1988) and Lu et al. (1992). These authors invoked parallel potential drops accelerating ions to 
explain the evolution of the ion-velocity distribution function with altitude. At an altitude of about $2 \mathrm{R}_{\mathrm{E}}$ at $2100 \mathrm{MLT}$ on the $70^{\circ}$ MLAT magnetic field line, fieldaligned $\mathrm{O}^{+}$ions with energy in the range $0.2-3 \mathrm{keV}$ were identified. Several mechanisms are plausible in the process of heating and/or acceleration of ionospheric ions. The perpendicular energy gained by the ion from ion cyclotron waves can be converted by the magnetic mirror force into parallel energy for upward motion. Parallel electrostatic fields, not balanced pressure gradient forces and ambipolar electric fields, can be the cause of further acceleration. The electric field induced by the magnetic-field time change may also accelerate the ions.

Ion characteristics measured in the near-geosynchronous regions were reported by, among others, Fennell et al. (1981) and Kaye et al. (1981). The ion velocity was predominantly field aligned at energies below a specific transition energy and predominantly perpendicular to the magnetic field at energies above. The transition energy varied in the range $2-10 \mathrm{keV}$, increasing during injection events. The low-energy component was composed primarily of $\mathrm{O}^{+}$and an ionospheric source was suggested for $\mathrm{O}^{+}$.

Plasma composition measurements made on-board the AMPTE/CCE satellite revealed how the plasma composition changes in the equatorial regions. The average energy of oxygen ions is of the order of tens of $\mathrm{keV}$ and, at active times, the phase-space density increases sharply in the energy range from 1 to $50 \mathrm{keV}$ (Kistler et al., 1989). The correlation between the composition of the equatorial plasma and the level of magnetic activity measured by auroral indices was reported by Daglis et al. (1993). During active times the abundance of $\mathrm{O}^{+}$and its contribution to the total energy density in the nightside magnetosphere increase much more than that of protons, especially at energies of tens of $\mathrm{keV}$. The $\mathrm{O}^{+}$population is field aligned and its energy density correlates well (correlation coefficient is 0.75) with the AE index during the substorm expansion phase (Daglis et al., 1994). In a case study, Daglis et al. (1990) presented and discussed AMPTE/CCE data from the postmidnight local-time sector. A fivefold enhancement of $\mathrm{O}^{+}$energy density just before substorm onset in a stretched magnetic field configuration and a twenty-five-fold increase a few minutes after the onset was recorded. The population was predominantly fieldaligned.

Baker et al. (1982), analyzing data recorded during substorm growth and expansion, suggested that asymmetries in the distribution of enhanced densities of $\mathrm{O}^{+}$might define regions of the plasma sheet in which the tearing mode instability threshold is lowered. The authors located the region in the range $x=-10--15 \mathrm{R}_{\mathrm{E}}$ and $y=5 \mathrm{R}_{\mathrm{E}}$.

Observations of the substorm magnetospheric field reveal the fluctuating nature of its large-scale changes during tail-like to dipole-like and reverse reconfigurations. Lui et al. (1992) identified magnetic-field reconfiguration events on AMPTE/CCE magnetograms by the occurrence of the magnetic-field fluctuations coinciding with ground onsets or intensifications of the westward auroral jet. The events were recorded at radial distance beyond the geosynchronous orbit: $7.4-8.8 \mathrm{R}_{\mathrm{E}}$ within a narrow north-south interval of $\Delta z=0.5 \mathrm{R}_{\mathrm{E}}$. The probability of detecting these events is high within $1 \mathrm{~h}$ of the local magnetic midnight. The duration time is of several (2.5-5) minutes. Events are manifested on the ground auroral magnetograms as the decrease in the horizontal component of the magnetic field monitoring the westward electrojet growth.

Observations of longer (over $10 \mathrm{~min}$ ) reconfiguration events in the south lobe at radial distances $17.5-22.6 \mathrm{R}_{\mathrm{E}}$ in the interval $\Delta z=7-10 \mathrm{R}_{\mathrm{E}}$ recorded on-board ISEE1 were presented and interpreted by Jacquey and Sauvaud (1994). Typical collapse of the field in the lobe distinguished by the authors as $B_{x}$ decrease accompanied by $B_{z}$ increase is nearly simultaneous with the auroral electrojet intensification and latitudinal movement of the auroral current seen on the ground magnetograms. The fine structure of the remaining "magnetic-field stretching" intervals is also discernible on the ground records. The reconfiguration events are linked with the cross-tail current dynamics, with its build-up resulting in field stretching during the substorm growth phase and with its disruption followed by the field collapse. The process in the tail coincides with auroral-electrojet intensification, making plausible the idea of current wedge formation.

A large $\left(30-\mathrm{mV} \mathrm{m}^{-1}\right.$ peak) westward electric field recorded on-board ISEE1 at radial distance $7.5 \mathrm{R}_{\mathrm{E}}$ postmidnight (0134 LT) at latitude $-20^{\circ}$ was reported by Aggson et al. (1983). Simultaneous collapse of the magnetic field was recorded by the satellite magnetometers. A one hundred-fold increase of $30-\mathrm{keV}$ in proton flux coincided with the auroral electrojet intensification.

Potential electric field was measured on-board the Viking satellite. In a statistical study of Lundqvist and Marklund (1990), the analysis of data from 109 duskdawn orbits was focused on the occurrence and properties of the two components of the electric field - parallel and transverse to $\boldsymbol{B}$. The parallel component is in general upward with an average value of $1 \mathrm{mV} \mathrm{m}^{-1}$. Its intensity depends on local time and increases with latitude and altitude. The perpendicular component maximizes $\left(5 \mathrm{mV} \mathrm{m}^{-1}\right)$ near local noon. Lower densities of plasma correspond to higher values of the electric field and the major part of the auroral oval is characterized by intense and irregular electric fields. The distribution of parallel electric field along the field line is an open problem.

The Earth's magnetosphere is time dependent; hence the necessity to include the induced electric field into the magnetospheric ion dynamics. Heikkila and Pellinen (1977) discussed the topic referring to substorm-time processes in the tail. These authors argue that the induced electric field parallel component in a magnetized plasma could not be canceled by the redistribution of charges but by the enhancement of the perpendicular component. Electric fields of various natures were studied by many authors (e.g. Hultqvist, 1988; Ganguli et al., 1994; Stern, 1981).

Ion motion in a time-dependent magnetic field with the induced electric field included was the subject of several studies. Mauk (1986) introduced an intense $\left(20 \mathrm{mV} \mathrm{m}^{-1}\right)$ equatorial electric field lasting $1 \mathrm{~min}$ transverse to the magnetic field collapsing from a stretched, tail-like 
configuration to a dipole-like one. The perpendicular and parallel components of motion were calculated under the assumption of the magnetic-moment conservation. The model successfully reproduced ion spectrograms sampled by the geosynchronous ATS-6 satellite. Protons and oxygen ions with different initial energies and pitch angles launched at different latitudes exhibit different behavior depending on the phase of their bounce motion during dipolarization. Ions very close to the equator during the event gain maximum energy. The isotropic predipolarization distributions split into parallel and perpendicular components. The final field-aligned (perpendicular) oxygen distributions peak between $10-20 \mathrm{keV}(2-4 \mathrm{keV})$. Final pitch-angle distributions depend on energy; oxygen ions populate the low pitch-angle interval.

The empirical model magnetosphere of Mead and Fairfield (1975) was used by Delcourt et al. (1990) to construct the time-dependent magnetospheric field and the induced electric field. The motion of ions launched at low latitudes at different sites $x<-9 \mathrm{R}_{\mathrm{E}}$ in the noonmidnight plane was investigated in detail, and the oscillatory changes of the magnetic moment were explained. Inspired by that study we followed the idea of Delcourt et al. (1990) and modified the fields model guided by the mentioned field data.

The objective of this study is to investigate the motion of the oxygen ion flowing out from the auroral ionosphere into the reconfigurating magnetosphere. The induced electric field is included in the equation of motion.

\section{Time-dependent field model, reconfiguration events}

\subsection{Magnetic field}

For its simplicity, the Mead-Fairfield magnetosphere model (Mead and Fairfield, 1975) is adopted in this study. It is an empirical model confined to the region determined by the radial distances $4-17 R_{E}$ in the equatorial plane. The components of the external field are represented by second-degree polynomials of space coordinates and by linear dependence on the tilt angle. Dawn-dusk and south-north symmetry of the external magnetic field and the zero-divergence condition are built into the model. Four sets of 17 coefficients, each referring to a specific magnetic activity level scaled by the $K p$ index, were derived by a least-squares method of data fitting. The magnetospheric field is the sum of the dipole field and the external field:

$$
\begin{aligned}
& \boldsymbol{B}=\boldsymbol{B}^{d}+\boldsymbol{B}^{\mathrm{ext}}, \\
& B_{x}^{\mathrm{ext}}= a_{1} Z+a_{2} X Z \\
&+T\left(a_{3}+a_{4} X+a_{5} X^{2}+a_{6} Y^{2}+a_{7} Z^{2}\right), \\
& B_{y}^{\mathrm{ext}}= b_{1} Y Z+T\left(b_{2} Y+b_{3} X Y\right), \\
& B_{z}^{\mathrm{ext}}= c_{1}+c_{2} X+c_{3} X^{2}+c_{4} Y^{2}+c_{5} Z^{2} \\
&+T\left(c_{6} Z+c_{7} X Z\right),
\end{aligned}
$$

where $a_{i}, b_{i}, c_{i}$ are $K p$ dependent. The space coordinates are given in units of ten Earth radii, tilt angle $T$ in units of ten degrees and $B$ in nanoteslas. A magnetospheric current derived by calculating $\nabla \times \boldsymbol{B}^{\text {ext }}$ is present in the model:

$$
\begin{aligned}
& j_{x}=\left(2 c_{4}-b_{1}\right) Y, \\
& j_{y}=\left(a_{1}-c_{2}\right)+\left(a_{2}-2 c_{3}\right) X+\left(2 a_{7}-c_{7}\right) T Z, \\
& j_{z}=\left(b_{3}-2 a_{6}\right) T Y .
\end{aligned}
$$

It follows from the formulae that for $T=0$, the $z$ component of the current vanishes. The projection of the current on $\boldsymbol{B}$ reproduces only qualitatively a region-1 Birkeland current upward in the dusk hemisphere, downward in the dawn hemisphere, though quantitatively the current density is a few orders of magnitude lower $\left(\sim 10^{-11} \mathrm{Am}^{-2}\right)$ than the real values.

Ding et al. (1994) investigated a series of other models and calculated the Birkeland current for each of them. Their result was that the region- 1 current pattern is reproduced from the tested models but that the values of the current are of the same order as those obtained from the Mead-Fairfield model.

Our intention is to study the details of ionospheric oxygen-ion motion in the magnetosphere reconfigurating in time from an initial state defined by the initial value $K p_{0}$ of the index to a final state with the final value $K p_{f}$. The two states are identified with the appropriate sets of coefficients and we assume the transition is not step-like and lasts $t_{d}$ seconds. We introduce time into the MeadFairfield magnetosphere following the idea of Delcourt et al. (1990):

$$
\boldsymbol{B}\left(\boldsymbol{r}, t_{0}+d t\right)=\boldsymbol{B}\left(\boldsymbol{r}, t_{0}\right)+f(t)\left[\boldsymbol{B}^{\mathrm{ext}}\left(\boldsymbol{r}, t_{f}\right)-\boldsymbol{B}^{\mathrm{ext}}\left(\boldsymbol{r}, t_{0}\right)\right] ;
$$

i.e. all the coefficients of the model are made time dependent by:

$c_{i} \rightarrow c_{i}(t): c_{i}(t+d t)=c_{i}\left(t_{0}\right)+f(t) \Delta c_{i}$,

$\Delta c_{i}=c_{i}\left(t_{f}\right)-c_{i}\left(t_{0}\right)$,

where $c_{i}\left(t_{0}\right)$ and $c_{i}\left(t_{f}\right)$ stand for the values of any coefficient of the model at the initial and final states determined by the $K p_{0}$ and $K p_{f}$ values; $t_{d}=t_{f}-t_{0} ; f(t)$ is a function of time such that $f\left(t_{0}\right)=0, f\left(t_{f}\right)=1$, and its rate of change is determined by the transition time $t_{d}$. The polynomial proposed for $f(t)$ by Delcourt et al. (1990) is adopted:

$f(t)=\beta_{5} \tau^{5}+\beta_{4} \tau^{4}+\beta_{3} \tau^{3}, \tau=t / t_{d}$,

where the coefficients were determined under additional conditions imposed on $f(t) ; \beta_{5}=6, \beta_{4}=-15, \beta_{3}=10$ (the reader is urged to consult that paper for details). Two fundamental reconfigurations can be simulated - collapse and stretching. We define a collapse as the transition between two states of the magnetosphere determined by the $K p$ values if $K p_{0}>K p_{f}$. A stretching is the reverse transition: the magnetosphere transforms from the quiet dipole-like state to a disturbed tail-like configuration. 


\subsection{Induced electric field}

The electric field induced by the nonzero $\partial \boldsymbol{B} / \partial t$ is derived from the relation:

$\boldsymbol{E}^{\text {ind }}=-\partial \boldsymbol{A} / \partial t$

where $\boldsymbol{A}$ is the vector potential of the external magnetic field and is scaled in the same way as $\boldsymbol{B}$ :

$\boldsymbol{A}(\boldsymbol{r}, t)=\boldsymbol{A}\left(\boldsymbol{r}, t_{0}\right)+f(t)\left[\boldsymbol{A}\left(\boldsymbol{r}, t_{f}\right)-\boldsymbol{A}\left(\boldsymbol{r}, t_{0}\right)\right]$.

The details of the derivation of the components of the vector potential are presented in Appendix A. It follows from the representation of the induced electric field by vector potential:

$\boldsymbol{E}^{\text {ind }}=-d f / d t\left[\boldsymbol{A}\left(\boldsymbol{r}, t_{f}\right)-\boldsymbol{A}\left(\boldsymbol{r}, t_{0}\right)\right]$

that:

- the field satisfies Faraday's law and the quasineutrality condition;

- the field amplitude at a point in space depends on the event duration $t_{d}$;

- the amplitude reaches the highest value at $t=t_{d} / 2$;

- the induced electric field has no $x$ component at midnight $(y=0)$.

The problem of balancing the parallel component of the induced $\boldsymbol{E}$ should be considered open. The electric field satisfies the quasi-neutrality condition $\nabla \boldsymbol{E}^{\text {ind }}=0$ and we will focus our attention in this study on the motion of ions in the reconfigurating magnetosphere with no potential terms added to amplify or attenuate the induced electric field.

\section{Results}

\subsection{Magnetic field}

In Fig. 1 (top), three auroral field lines $65^{\circ}, 68^{\circ}$, and $70^{\circ}$ MLAT in the noon-midnight plane are plotted for two states of the MF magnetosphere: superquiet, $K p=0$, and superdisturbed, $K p>3$. The small difference between the two states at the dayside expands later in local time to larger values; at midnight the distance between the equatorial crossing points of the $70^{\circ}$ MLAT field line for the two states is about $5 \mathrm{R}_{\mathrm{E}}$. The surface spanned by the magnetic field lines anchored on the Earth at an MLAT cross the equatorial plane along a closed curve, the shape and the position of which depend on MLAT and on the state of magnetosphere. The curves are represented by dots and crosses for quiet and disturbed magnetosphere, respectively, in Fig. 1 (bottom). The region enclosed with the curves corresponding to two different states of the magnetosphere increases sharply with MLAT reaching the area of about $95 \mathrm{R}_{E}^{2}$ for the $70^{\circ}$ MLAT lines. At 1 -h-MLT, $1^{\circ}$-MLAT-wide auroral region is mapped to the equatorial region of the area depending on local time. During a reconfiguration event the $70^{\circ}$ lines move over the longest radial distances in the local evening at

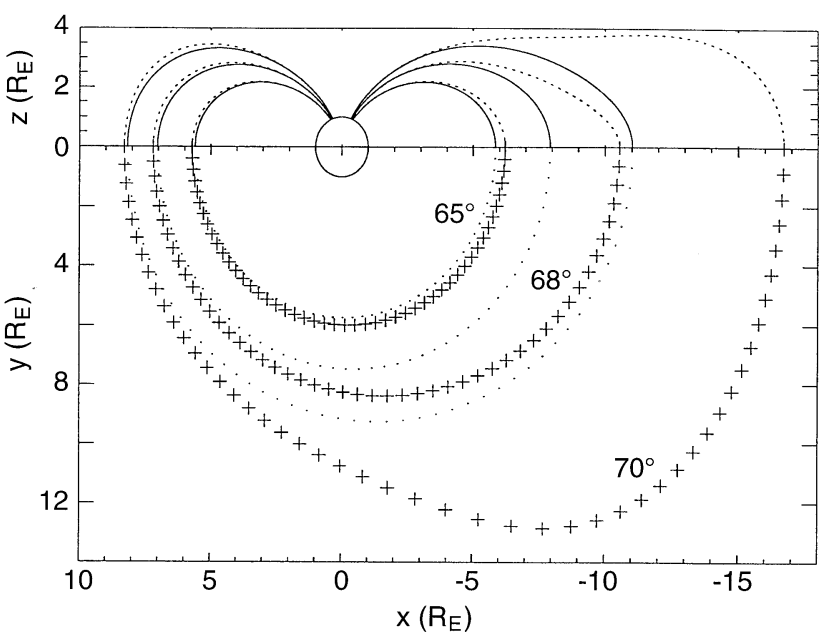

Fig. 1. Auroral field lines $\left(65^{\circ}, 68^{\circ}\right.$, and $70^{\circ}$ MLAT) of the MeadFairfield magnetosphere model. In the noon-midnight plane (top) quiet state is represented by continuous lines and the disturbed state by dotted lines. The field-line footprints in the equatorial plane (bottom) are represented by dots for quiet state and by crosses for the disturbed magnetosphere

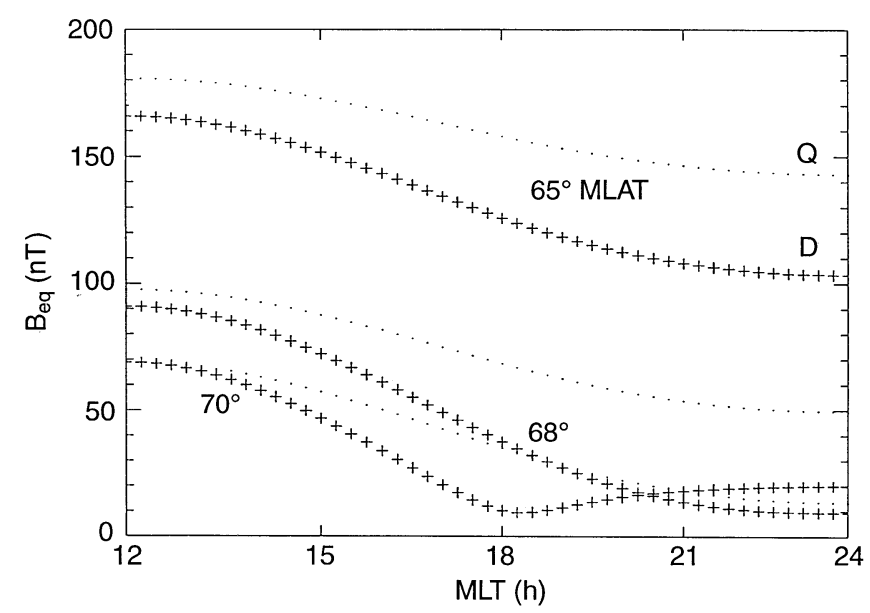

Fig. 2. Magnetic-field intensity at the equatorial plane versus local time for $65^{\circ}, 68^{\circ}$, and $70^{\circ}$ MLAT field lines for quite (dots) and for disturbed (crosses) magnetosphere

1800 MLT. $B_{e q}$, the magnetic-field intensity at the equatorial crossing points of the field lines, is plotted versus local time for quiet and disturbed conditions in Fig. 2. For $65^{\circ}$ and $68^{\circ}$ lines the relation is monotonous. $B_{e q}$ exhibits a minimum for the disturbed $70^{\circ}$ lines at $\sim 1800$ MLT. This implies the dependence of the cyclotron period on local time: for ions moving along the $70^{\circ}$ MLAT line the maximum cyclotron period near the equatorial plane is at 1800 MLT.

The current present in the MF magnetosphere for $T=0$ is a planar vector field with no $z$ component, linearly dependent on the $X, Y$ coordinates. The fieldaligned current density changes along the field line. The region where it reaches the maximum value is defined by the planar nature of the current field and its space variability. The field-aligned current reaches its maximum 
value not far from the point where the magnetic field line reaches its maximum $z$ component. In the MF magnetosphere the upward current in the northern hemisphere is most intense at 1800 MLT. There are no currents at noon and at midnight. The polarization electric field, proportional to $d \boldsymbol{j} / d t$ and weighted with the plasma cyclotron frequencies (Ganguli et al., 1994) is downward in the dusk hemisphere. Its values support the assumption that it can be neglected even if one adopts the real values for the field-aligned current intensity.

\subsection{Induced electric field}

The coefficients of the vector potential difference defining the induced electric field in Eq. 8 are as follows: for $A_{x}$, $\Delta b_{1}= \pm 8.99 \mathrm{nT} /\left(10 \mathrm{R}_{\mathrm{E}}\right)^{2}, \Delta c_{4}=\mp 7.18 \mathrm{nT} /\left(10 \mathrm{R}_{\mathrm{E}}\right)^{2}$, for $A_{y}, \Delta a_{1}=\mp 21.55 \mathrm{nT} / 10 \mathrm{R}_{\mathrm{E}}, \Delta a_{2}=\mp 2.88 \mathrm{nT} /\left(10 \mathrm{R}_{\mathrm{E}}\right)^{2}$, $\Delta c_{1}= \pm 13.49 \mathrm{nT}, \Delta c_{2}=\mp 7.63 \mathrm{nT} / 10 \mathrm{R}_{\mathrm{E}}, \Delta c_{3}=\mp 13.34$ $\mathrm{nT} /\left(10 \mathrm{R}_{\mathrm{E}}\right)^{2}$. The plus- or- minus signs are for collapse and stretching, respectively. Then the components of the electric field $\left(X, Y, Z\right.$ in tens of $\left.\mathrm{R}_{\mathrm{E}}\right)$ are: $E_{x}^{\text {ind }}=-d f / d t$ $\left(\Delta b_{1} Y Z^{2}-\Delta c_{4} Y^{3} / 3\right), E_{y}^{\text {ind }}=-d f / d t\left(-\Delta a_{1} Z^{2} / 2-\right.$ $\left.\Delta a_{2} X Z^{2}+\Delta c_{1} X+\Delta c_{2} X^{2} / 2+\Delta c_{3} X^{3} / 3\right)$. The inducedelectric-field variability in space is determined by the vector potential dependence on spatial variables. On the Sun-Earth line $(Y=0, Z=0)$ at the nightside, the field is westward for collapse and eastward for stretching. During collapse at $1800 \mathrm{MLT}(X=0)$ in the equatorial plane $(Z=0)$ the field is eastward; at 0600 MLT it is westward.

The time dependence of the induced electric field at a given point in space is defined by the value of the time-derivative of the function $f(t)$, which depends on the duration time of the reconfiguration event:

$d f / d t=t_{d}^{-1}\left(5 \beta_{5} \tau^{4}+4 \beta_{4} \tau^{3}+3 \beta_{3} \tau^{2}\right)$,

$(d f / d t)_{\max }=t_{d}^{-1}(d f / d t)\left(t=t_{d} / 2\right)=1.87 t_{d}^{-1}$.

The charged-particle motion is determined by the components of the electric field parallel and perpendicular to the magnetic field. The maxima along the magnetic field line of these components for the 5-min collapse at the moment $t=t_{d} / 2$ are plotted versus local time in Fig. 3 for three magnetic field lines of different latitude and for northern hemisphere. The maxima are attained at a point of the field line dependent on the line latitude and on the fact that the vector potential is an increasing function of the distance from the Earth. The dependence is seen in Fig. 4, where the isolines of the parallel and perpendicular components in the 2100-MLT meridian plane are plotted. It follows from the dependence on local line (Fig. 3) that the polarity of the parallel induced electric field is distributed in four sectors of local time with north-south asymmetry; in the north, the upward (with respect to $\boldsymbol{B}$ ) field is localized in 18-24 and 06-12 local-time sectors, the downward field is present in postmidnight and postnoon hours. The $\mathrm{max} / \mathrm{min}$ value of the field-aligned component of $\boldsymbol{E}^{\text {ind }}$ is attained in the middle of the sector and is a monotonous function of the magnetic field line latitude reaching values of $\sim 6-7 \mathrm{mV} \mathrm{m}^{-1}$ on the $70^{\circ}$ MLAT line.

The perpendicular component of the induced electric field has different local-time dependence. Its values are low at dawn and at dusk. Near noon the value is $\sim 15 \mathrm{mV} \mathrm{m}^{-1}$. At midnight the perpendicular electric field intensity is about $7 \mathrm{mV} \mathrm{m}^{-1}$.

The drift velocity $V_{D}=\left[E_{y} B_{z},-E_{x} B_{z}, E_{x} B_{y}-E_{y} B_{x}\right] / B^{2}$ is an increasing function of radial distance ranging from zero value at the beginning of the event up to $7 \mathrm{mV} \mathrm{m}^{-1} / 20 \mathrm{nT}=350 \mathrm{~km} \mathrm{~s}^{-1}$ at the moment of half-collapse. Along the Sun-Earth line $(y=0, z=0)$, the drift is earthward during a collapse event and tailward during stretching. In the noon-midnight meridian plane, at the point of the field line where $B_{z}=0, V_{D}$ has only the $z$-component and is equatorward.

It follows from the described topology of the reconfigurating magnetic field and of the induced electric field, that the effective extraction of the ionospheric UFIs from the ionosphere and their subsequent movement into other regions of the magnetosphere is determined by the azimuthal dependence of the magnetic field line geometry

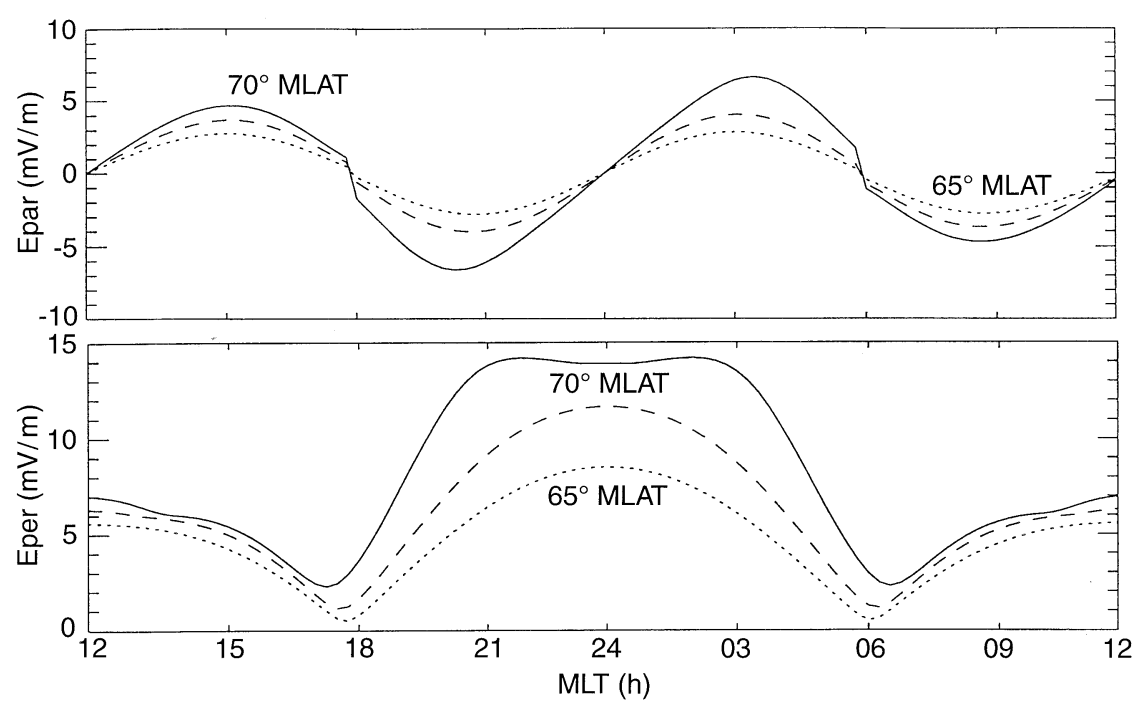

Fig. 3. The intensity of field-aligned and perpendicular components of the induced electric field is calculated along field lines at half-collapse $\left(t=t_{d} / 2\right)$. The maximum value in the northern hemisphere is found and plotted versus local time; top-field-aligned, bottomperpendicular component; $65^{\circ}$ (dotted), $68^{\circ}$ (dashed), and $70^{\circ}$ (continuous) MLAT field lines are considered. In the sourthern hemisphere the polarity of the field-aligned component is reversed; in the 18-24-MLT sector it is downward the magnetic field 

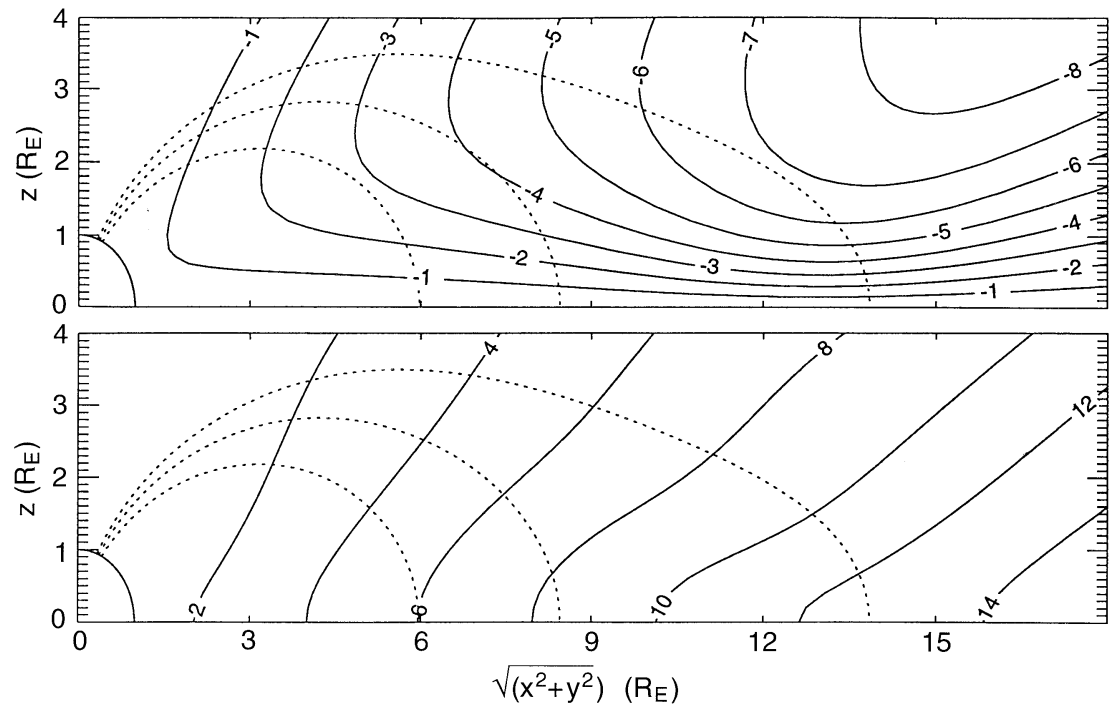

Fig. 4. The isolines of the intensity of the field-aligned (top) and perpendicular (bottom) components of the induced electric field in the 2100-MLT meridian plane at the moment of half-collapse $\left(t=t_{d} / 2\right)$. The $65^{\circ}, 68^{\circ}$, and $70^{\circ}$ MLAT magnetic field lines are overplotted and of the two components of the electric field. In a collapse case the equatorial regions can be populated either by the ions coming from the north in the 18-24 MLT hours or by those coming from the south. Intense perpendicular electric field (up to $15 \mathrm{mV} \mathrm{m}^{-1}$ ) reaching the maximum in the equatorial plane will affect the perpendicular component of the motion.

\section{Oxygen-ion motion}

The full equation of motion

$\boldsymbol{r}^{\prime \prime}=q / m\left(\boldsymbol{E}^{\text {ind }}(\boldsymbol{r}, t)+\boldsymbol{r}^{\prime} \times \boldsymbol{B}(\boldsymbol{r}, t)\right)$,

is solved for $\mathrm{O}^{+}$ions; here $\boldsymbol{r}$ is the particle position vector; $q$ and $m$ are the particle charge and mass, respectively; $\boldsymbol{B}(\boldsymbol{r}, t)$ is the magnetic field and $\boldsymbol{E}^{\text {ind }}(\boldsymbol{r}, t)$ is the induced electric field. The ions are launched at the altitude of $2 \mathrm{R}_{\mathrm{E}}$ at $65^{\circ}-71^{\circ} \mathrm{MLAT}$ in the $1800-2400$-MLT sector. The initial energy $\left(\varepsilon_{i}\right)$ range is $0.3-3.2 \mathrm{keV}$ and the initial pitchangle $\left(p a_{i}\right)$ range is $90^{\circ}-180^{\circ}$. The equation is integrated using the fourth-order Runge-Kutta procedure during a 5-min collapse event already defined as the transition from an elongated tail-like configuration $(K p>3 \mathrm{MF}$ magnetosphere) to a near dipole state $(K p=0)$. At any point in space the maximum value of the induced electric field is in inverse proportion to $t_{d}$. For longer events the field is weaker everywhere and the motion lasts longer. We consider $t_{d}=5 \mathrm{~min}$ as the intermediate value between the short, 1-min events, which may be local in space, and the substorm-scale long events (tens of minutes). The attention will be focused on the characteristics of the ions attained in the near-equatorial regions and on the details of the acceleration process.

\subsection{Characteristics of ions at the equatorial plane}

In Fig. 5a the energy of the $\left(3.2-\mathrm{keV}, 180^{\circ}\right)$ oxygen ion attained on the equatorial plane is plotted as a function of
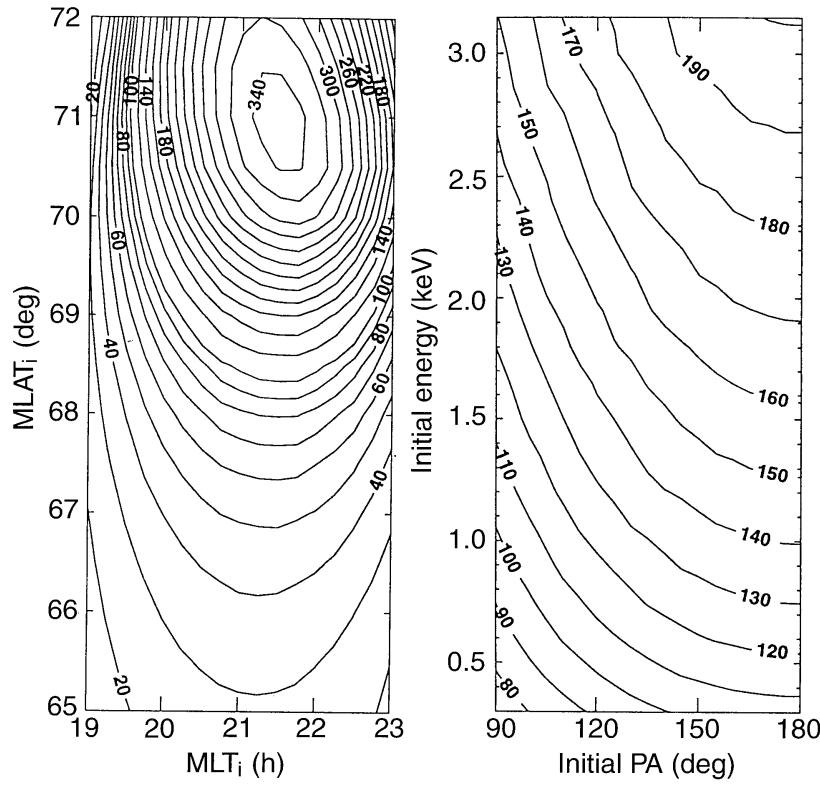

Fig. 5. The isolines of the equatorial energy of the $3-\mathrm{keV}, 90^{\circ}$ ion vs local time and latitude of launching (left) and the equatorial energy of ions launched at 2100 MLT, 69 MLAT as a function of ion initial energy and pitch angle (right). The isolines are labeled with energy in $\mathrm{keV}$

$\left(\mathrm{MLAT}_{i}, \mathrm{MLT}_{i}\right)$. The greatest increase in energy is for ions ejected from a particular region: $\operatorname{MLAT}_{i} \sim 71^{\circ}$, $\mathrm{MLT}_{i} \sim 2130$. The position of the energy maximum follows from the dependence of the $E_{\|}^{\text {ind }}$ on local time, its maximum is near $2100 \mathrm{MLT}$, and from the increase in the electric-field intensity with the radial distance. Longer parts of the $70^{\circ}-71^{\circ}$ MLAT lines pass the regions where the intensity of electric field is high compared with the $65^{\circ}-68^{\circ}$ lines penetrating regions with the electric field of lower intensity, see Fig. 4. Figure 5b illustrates the dependence of the equatorial energy on the ion initial energy and pitch angle for ions launched at $69^{\circ}$ MLAT, 2125 MLT. Field-aligned ions are most energized. 


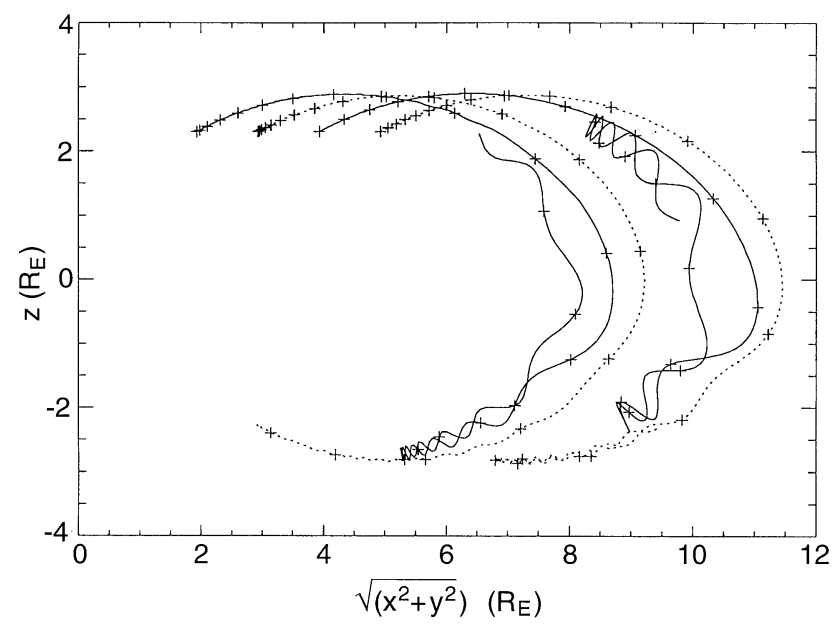

Fig. 6. The trajectories of ions as projected onto the 2125-MLT meridian plane. Ions are launched along the $68^{\circ}$ MLAT magnetic field line. All but the $3-\mathrm{keV}, 90^{\circ}$ trajectory are shifted to the right for legibility by 1,2 , and $3 \mathrm{R}_{\mathrm{E}}$ for $300-\mathrm{eV}, 90^{\circ} ; 3-\mathrm{keV}, 180^{\circ} ; 300-\mathrm{eV}, 180^{\circ}$ ions, respectively. $300-\mathrm{eV}$ ion trajectories are represented by dotted, $3-\mathrm{keV}$ ions by continuous curves

\subsection{Acceleration details}

The acceleration will be discussed for four ions: low and high energy, perpendicular and field aligned: $\left(300 \mathrm{eV}, 90^{\circ}\right)$, $\left(300 \mathrm{eV}, 180^{\circ}\right),\left(3 \mathrm{keV}, 90^{\circ}\right)$ and $\left(3 \mathrm{keV}, 180^{\circ}\right)$. Ions are launched at $68^{\circ}$ MLAT, 2125 MLT. The trajectories, ion energy and pitch-angle changes are plotted in Figs. 6 and 7 , and the magnetic-moment, cyclotron period and elec- tric-field components are plotted versus time in Fig. 8. The $3-\mathrm{keV}, 180^{\circ}$ ion (third from the left in Fig. 6) crosses after $146 \mathrm{~s}$, at an angle of $150^{\circ}$, the equatorial plane driven by a $4.28-\mathrm{mV} \mathrm{m}^{-1}$ electric field encountered $2 \mathrm{~s}$ earlier. Ion energy at the point $(x, y, z)=(-6.5,6.3,0) \mathrm{R}_{\mathrm{E}}$ is $71 \mathrm{keV}$; 10 s later at $z=-1.01 \mathrm{R}_{\mathrm{E}}$, its energy is maximum: $83 \mathrm{keV}$. The ion mirrors at $197 \mathrm{~s}$, and $30 \mathrm{~s}$ later recross the equatorial plane; its energy at the point $(x, y, z)=$ $(-4.26,6.72,0) \mathrm{R}_{\mathrm{E}}$ is $74 \mathrm{keV}$ and pitch angle is $24^{\circ}$; the drift is westward to the dayside. The $300-\mathrm{eV}, 90^{\circ}$ ion in its halfbounce motion reaches the equatorial plane within $214 \mathrm{~s}$, and $4 \mathrm{~s}$ later its energy is maximum: $59.2 \mathrm{keV}$, pitch angle is $160^{\circ}$ (at $z=-0.53 \mathrm{R}_{\mathrm{E}}$ ). The other two ions cross the equatorial plane at an angle from the $30^{\circ}$ cone around $\boldsymbol{B}$ and reach maximum energy in the regions below the equatorial plane as well. At the end of the event, the $300-\mathrm{eV}, 90^{\circ}$ ion reaches a latitude (see Fig. 6) where it encounters magnetic field of the intensity higher than at the start. The $300-\mathrm{eV}, 180^{\circ}$ ion mirrors at lower latitudes. The ion's motion is determined both by the electric-field spatial distribution and by its evolution in time (see Fig. 8, bottom). The $300-\mathrm{eV}, 90^{\circ}$ ion encounters the electric field of $2.86 \mathrm{mV} \mathrm{m}^{-1}$ at $202 \mathrm{~s}$; i.e., $52 \mathrm{~s}$ later from the moment of maximum field intensity (the moment of half-collapse) at the point, and reaches the equatorial plane when the field disappears $(214 \mathrm{~s})$. It gains less energy than the $3-\mathrm{keV}$, $180^{\circ}$ ion reaching the equatorial plane at the moment of the highest intensity of the electric field (146 s). A particular combination of ion initial characteristics and the reconfiguration event time-scale determine the ion trajectory, energy and pitch-angle changes. Low-energy ions miss the maximum electric field in the near-equatorial
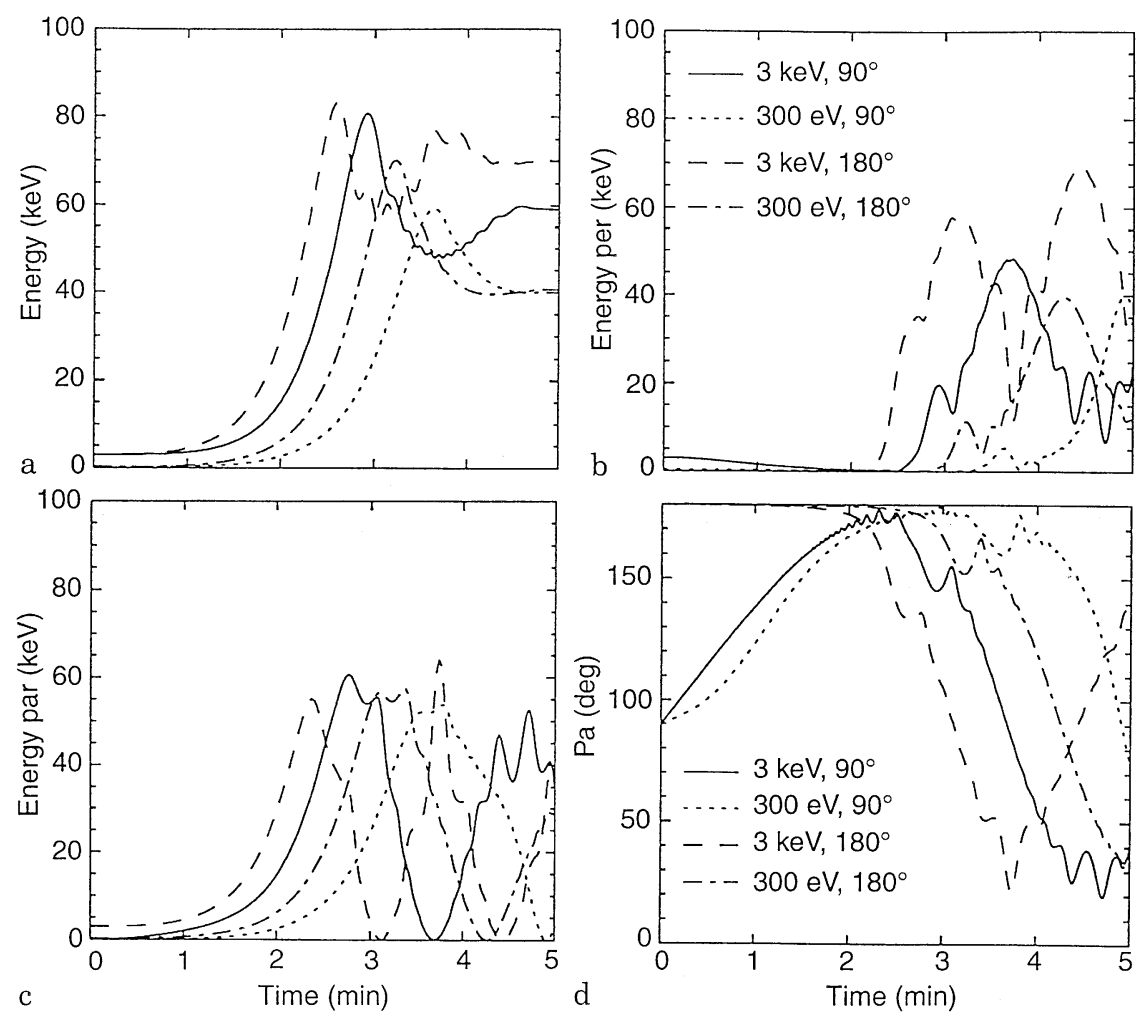

Fig. 7a-d. The details of ions motion. a Total energy; b perpendicular energy; c parallel energy; $\mathbf{d}$ pitch angle versus time 

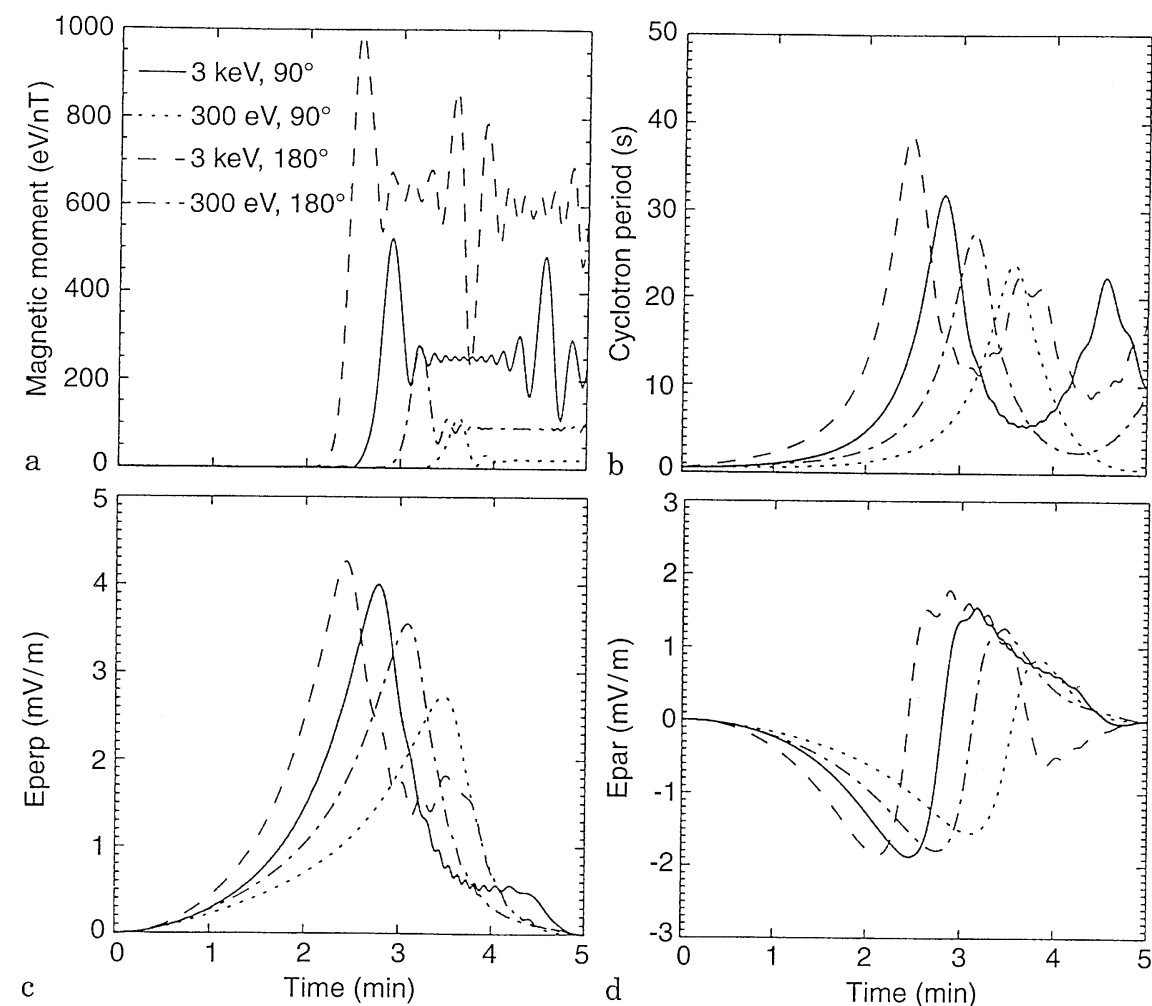

Fig. 8a-d. The details of ions motion.

a Magnetic moment; b cyclotron period, and c, $\mathbf{d}$ induced electric field components versus time
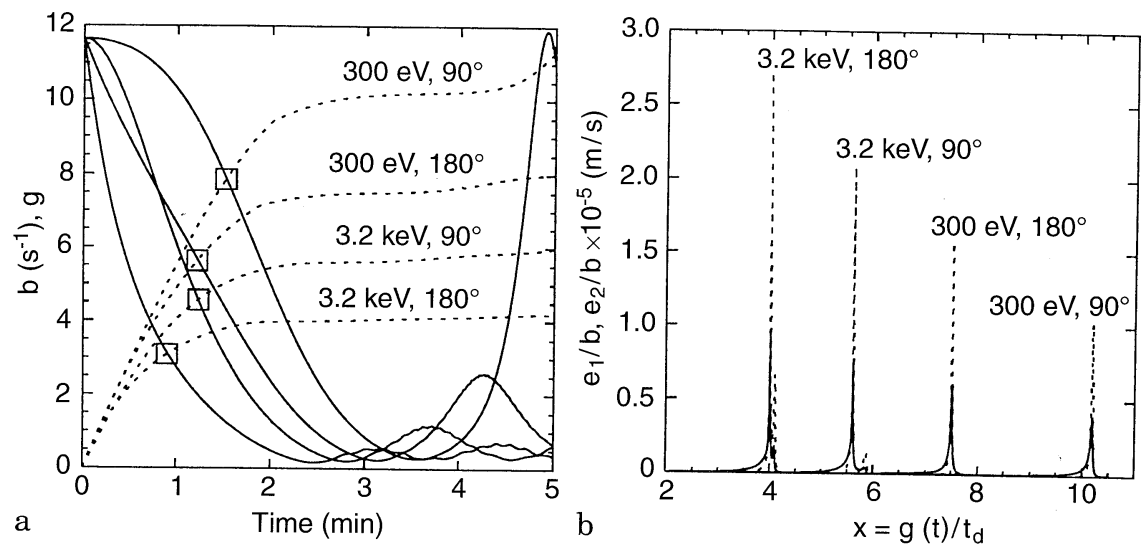

Fig. 9. a $b(t)=q / m B(t), g(t)=\int b(t) d t$ vs time. The dotted curves of $g(t)$ are assigned to the corresponding curves of $b(t)$ by squares. $\mathbf{b} e_{1} / b$ (continuous), $e_{2} / b$ (dotted) versus $x=g(t)$

regions. The 60-s initial phase and 40-s final phase of the motion when energy is conserved are separated by the phase of energy change. The oscillating nature of the energy change and oscillation of the pitch angle are characteristic for the near-equatorial stage of the $3-\mathrm{keV}, 90^{\circ}$ ion motion.

In the third minute of the motion, as the $3-\mathrm{keV}, 90^{\circ}$ ion approaches the point of the minimum magnetic-field intensity, its cyclotron period is $\sim 30 \mathrm{~s}$ and the perpendicular component of the electric field changes from the value $1.4 \mathrm{mV} \mathrm{m}^{-1}$ at $120 \mathrm{~s}$ to $4 \mathrm{mV} \mathrm{m}^{-1}$ at $168 \mathrm{~s}$ (Fig. 8). There is a jump of the magnetic moment $\Delta \mu=340$ (at $168 \mathrm{~s})-1.62$ (at $120 \mathrm{~s}$ ) $=338.38 \mathrm{eV} \mathrm{nT}^{-1}$, followed by oscillations at the level of $230 \mathrm{eV} \mathrm{nT}^{-1}$. For the $300-\mathrm{eV}, 90^{\circ}$ ion, the growth rate of the electric field is a factor of
2 lower, as $\Delta E_{\perp}=E_{\perp}(t=202 \mathrm{~s})-E_{\perp}(t=120 \mathrm{~s})=2.87-$ $0.6=2.27 \mathrm{mV} \mathrm{m}^{-1}$ and the jump of the magnetic moment is $\Delta \mu=81 \mathrm{eV} \mathrm{nT}^{-1}$.

In the time-dependent field $\Delta \mu=\left(\varepsilon_{\perp} / B\right)^{\prime} \Delta t$. Neglecting the $\varepsilon_{\perp} B^{\prime} / B^{2}$ term, and applying the equation of perpendicular motion, one obtains $\Delta \mu=\Delta t \varepsilon_{\perp}{ }^{\prime} / B=\Delta t q \boldsymbol{E}_{\perp}(t) \boldsymbol{v}_{\perp} / B$ (see, e.g., Chen, 1984). The magnetic-moment change is in proportion to $\boldsymbol{E}_{\perp}(t)$, to $\boldsymbol{v}_{\perp}$, and to their relative orientation. Precise considerations allow to elucidate the change in detail; see Appendix B. The quantities $b(t)=q / m B(t)$, $x=g(t)=\int b(t) d t,\left(e_{1,2} / b\right)(x)=E_{1,2} / B(x)$ emerging while deriving the formula for $\mu(t)$ are plotted in Fig. 9. Two minutes after the start, the rate of change of the function $g(t)$, which the magnetic-moment oscillation frequency depends on, is higher for the $300-\mathrm{eV}$ ion than for the $3-\mathrm{keV}$ 

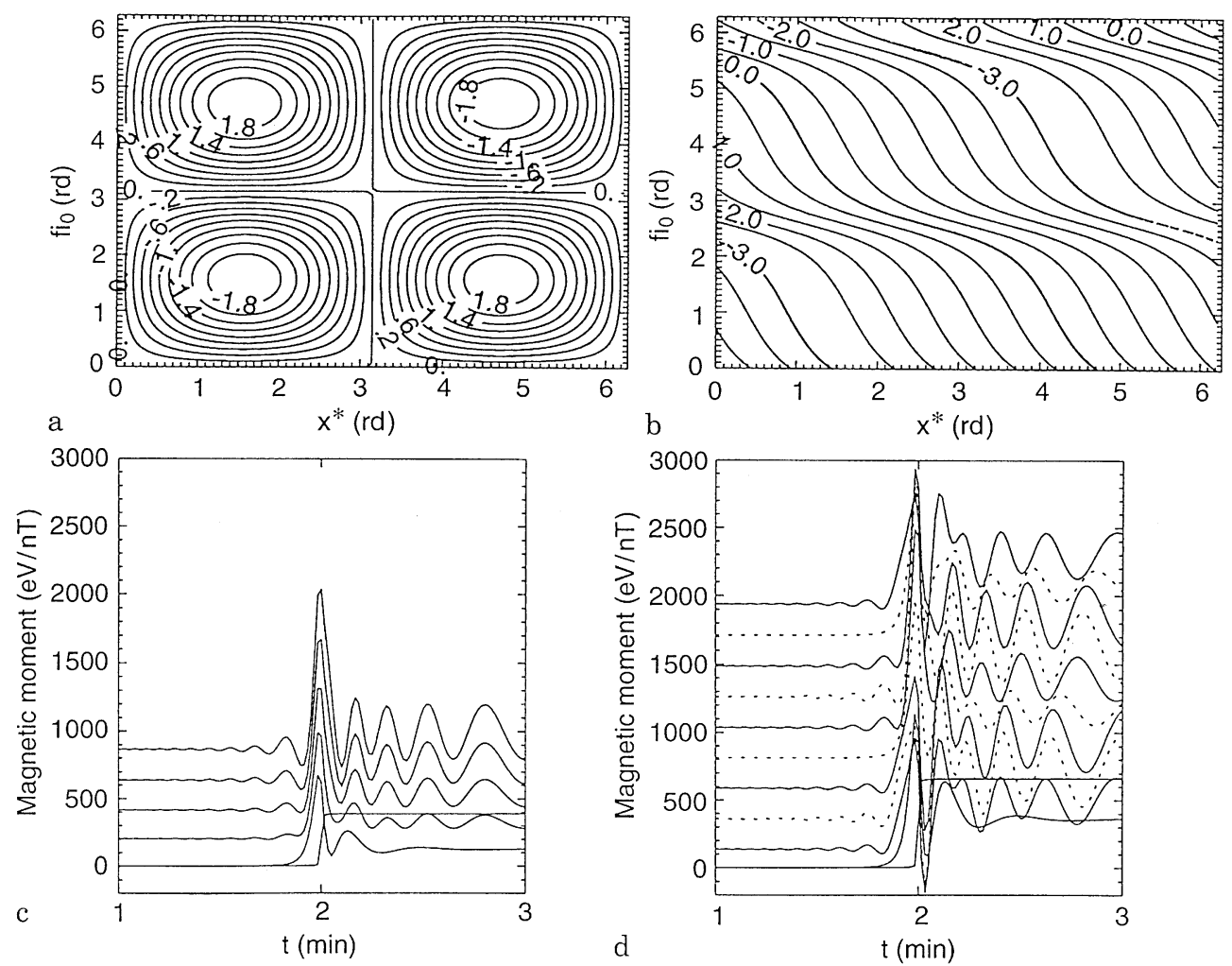

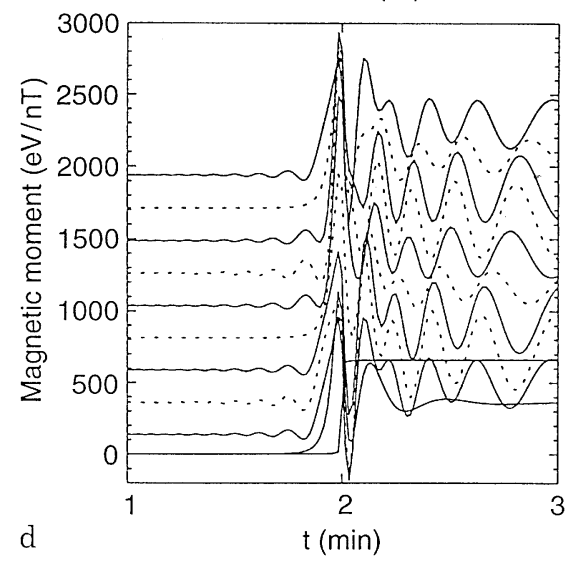

Fig. 10a-d. Ion magneticmoment dependence on time. The coefficients a $s_{0}$ and $\mathbf{b} s_{3}$ of Eq. 11 as functions of $x^{*}=g\left(t^{*}\right)$ and of $\varphi$ - the initial phase of the ion. The magnetic moment as function of time for two different values of the constant $\mathrm{A}$ : c $390 \mathrm{rd} \mathrm{km} \mathrm{s}^{-1}$, d $660 \mathrm{rd} \mathrm{km} \mathrm{s}^{-1}$. In $\mathbf{c}, v_{0}=0,100,200,300$, $400 \mathrm{~km} \mathrm{~s}^{-1}, \varphi=1.25 \pi$ d $v_{0}=300 \mathrm{~km} \mathrm{~s}^{-1}, \varphi=n$ $0.017 \pi, n=0,1, \ldots, 9$. In each panel the curves are shifted up the multiple of 200 units with respect to the $v_{0}=0$ and $n=0$ curves, for legibility ion. The delta-like shape of $e_{2} / b$ as a function of $x$ is obvious.

Let us consider, in general, the magnetic-moment change of an ion moving in time-dependent magnetic and electric fields, such that $e_{1} / b=e_{2} / b=A \delta\left(x-x^{*}\right)$, $\delta\left(x-x^{*}\right)$ is the delta function. The magnetic moment as function of time is represented by the expression:

$$
\begin{aligned}
\mu(t)= & m / 2 B\left\{v_{\perp 0}^{2}+2 V_{D}^{2}+2 A^{2} H^{2}\left(x-x^{*}\right)\right. \\
& +v_{0} A H\left(x-x^{*}\right) s_{0}\left(\varphi, x^{*}\right)-4 v_{0} V_{D} s_{1}(\varphi) \sin (\pi / 4+x) \\
& -4 V_{D} A H\left(x-x^{*}\right) \sin \left(x-x^{*}\right) \\
& \left.+v_{0} A H\left(x-x^{*}\right) s_{2}(\varphi) \sin \left(2 x+s_{3}\left(\varphi, x^{*}\right)\right)\right\}, x=g(t) .
\end{aligned}
$$

In Fig. 10 a, b the isolines of $s_{0}\left(\varphi, x^{*}\right)$ and $s_{3}\left(\varphi, x^{*}\right)$ are plotted. The values of $s_{0}$ are from the interval $[-2,2], s_{3}$ range is $[-\pi, \pi]$. The first term in the first line of Eq. 11 is the initial magnetic moment. The second term represents the drift-velocity, $V_{D}$, dependence on time resulting from the electric- and magnetic-field evolution in time (see, e.g., Fig. 8). This term determines the growth and the decrease of the magnetic moment. The Heavyside function simulates the jump of the magnetic moment (third and fourth terms) and puts on the oscillations following the jump. The jump is proportional to $2 A^{2}+v_{0} A s_{0}\left(\varphi, x^{*}\right)$. The amplitude and the frequency of the magnetic-moment oscillations are determined by three remaining terms. Oscillations with amplitude $4 v_{0} V_{D} s_{1}(\varphi)$ die out as $V_{D}$ decreases. Two other terms represent oscillations at the increased level of the magnetic moment. It follows from the formula that: firstly, there is no oscillation at the upper level of the magnetic moment if $v_{0}=0$; secondly, the amplitude and the frequency of the oscillation at the upper level are determined by the superposition of the last two terms. Figure $10 \mathrm{c}$, d illustrates how the quantities $v_{0}$, $V_{D}, A$, and $\varphi$ control the magnetic-moment changes. The two panels are for two values of the constant $A, x^{*}$ is put equal $\pi, B=30 \mathrm{nT}$. The jump $A H\left(x-x^{*}\right)$ is localized in the middle of the 2-min interval. The magnetic-moment level after the jump is represented by the very bottom curve, for which $v_{0}=0$, and hence no oscillation follows the jump. $\boldsymbol{V}_{D}(t)$ is simulated with $a / \pi /\left(a^{2}\left(t-t^{*}\right)^{2}+1\right)$, with $a=30, g(t)$ with $3 t_{d} \tanh (t)$. The frequency of oscillations $d g / d t=b(t)=0.5 \mathrm{~s}^{-1}$. In Fig. 10c the ion initial phase is kept constant, $\varphi=1.25 \pi$, and the oscillation-amplitude increase with $v_{0}$ is illustrated. In Fig. 10d it is shown how the initial phase affects the oscillations for constant $v_{0}=300 \mathrm{~km} \mathrm{~s}^{-1}$.

So, coming back to Fig. 8, the differences between the four ions in the magnetic-moment behavior can be explained. For the $300-\mathrm{eV}$ ions the value of $A$ is less then for the $3-\mathrm{keV}$ ions, and the magnetic-moment jump is lower. The energy of the $300-\mathrm{eV}, 180^{\circ}$ ion is the parallel component. Hence there is no oscillation at the upper level of the magnetic moment. For the $3-\mathrm{keV}$ ions the magnetic-moment jumps to the level of 250 and $600 \mathrm{eVnT}^{-1}$ and the amplitude of oscillations is proportional to the perpendicular energy. The oscillation frequency varies from $0.4 \mathrm{~s}^{-1}$ for the $3-\mathrm{keV}, 180^{\circ}$ ion to $0.8 \mathrm{~s}^{-1}$ for the $3-\mathrm{keV}$, $90^{\circ}$ ion. 


\section{Discussion and conclusions}

Reconfigurations of the magnetosphere during growth and expansion phases of substorm induce the electric field. Reconfigurations may be simulated by time-dependent models of the magnetosphere. In the study a time-dependent model of the magnetosphere was built by introducing time into the Mead-Fairfield empirical model of the magnetosphere. There is no reason to expect the induced electric field to be uniform in space. It follows from the spatial distribution of the induced electric field that there exist local-time sectors with reverse polarity of the fieldaligned component of the electric field. It implies azimuthal dependence of ionospheric ions supply to the equatorial regions. The intensity of the electric field is an increasing function of the distance from the Earth. It may be associated with the high-amplitude electric field measured by satellites. The intensity of the constructed electric field is inversely proportional to the reconfiguration event duration. The perpendicular component of the electric field maximizes near noon and at midnight. The structure of the induced electric field results from a particular choice of the vector potential and the aim was to illustrate the details of this structure. The real topology of the induced electric field is not known and we want to emphasize the hypothetical nature of the induced electric field adopted in the study.

The induced electric field is strong enough to energize the ions supplied by the ionosphere. The details of the motion; energy, pitch angle, mirror points and drift, depend on the ion initial characteristics and on the MLT, MLAT of the region of launching. Ions may be energized up to the $100-\mathrm{keV}$ energy level. The maximum energy is attained in the near-equatorial regions as the result of maximum intensity electric field. As the adiabaticity criterion is not satisfied, the magnetic moment is not conserved. The changes in the magnetic moment are determined by the initial velocity of the ion and by the spatial- and time-scales of the magnetic and induced electric fields defined by the reconfiguration-event parameters.

Charge separation effects and self-consistency were not addressed in the study; these problems were investigated by Mauk (1989), where the generation of the scalar potential of a purely field-aligned electric field is simulated. The model is made up of following assumptions: 1 . a magneticfield flux tube, filled with plasma and closed, reshapes during a 1-min dipolarization event; 2. a step-like electric field is put on for the period of event; 3 . the velocity distribution of electrons is bi-Maxwellian and time independent; 4 . the potential is derived imposing the quasineutrality condition; 5 . ions are traced self-consistently integrating the equation of the field-aligned motion and conserving the magnetic moment. Temporal and spatial characteristics of the evolving field-aligned electric field are ion-mass sensitive and depend on the temperature of electron and ion components of plasma. In case of oxygen ions and electrons of $\mathrm{kT}=1 \mathrm{keV}$, the intensity of the field-aligned electric field is about $0.1 \mathrm{mV} \mathrm{m}^{-1}$. As the field is antiparallel to $\boldsymbol{B}$ in the northern hemisphere, it would drive the extraction of ions from the ionosphere if the flux tube was open. So the field-aligned electric field resulting from the self-consistent calculus would not attenuate the field-aligned component of the induced electric field in the 18-24-MLT sector. Suitable measurements would verify the conclusions. Further studies are needed to elucidate the role the electric fields of different type play in the dynamics of ions during the substorm reconfigurations of the Earth magnetosphere.

Acknowledgements. The authors are grateful to the referees of this paper for their helpful suggestions. The study was supported by the Polish Committee of Scientific Research, grant 662299203.

The Editor-in-chief thanks I. A. Daglis and another referee for their help in evaluating this paper.

\section{Appendix A}

In choosing the form of the vector potential we were guided by the fact, mentioned earlier, that for $T=0$ we have $j_{z}=0$, so the $A_{z}$ component should also vanish. As the azimuthal dependence of many phenomena linked with magnetospheric disturbances (fieldaligned currents, field-aligned potentials, injection events, and electrojets) is still not measured and studied enough, we find it inappropriate to impose further restriction on the potential choice. So assuming $A_{z}^{\text {ext }}=0$ we obtain from the relation $\nabla \times \boldsymbol{A}^{\text {ext }}=\boldsymbol{B}^{\text {ext }}$ the following expressions:

$$
\begin{aligned}
A_{x}= & b_{1} Y Z^{2} / 2+T\left(b_{2} Z Y+b_{3} X Y Z\right)+g(X, Y), \\
A_{y}= & -a_{1} Z^{2} / 2-a_{2} X Z^{2} / 2-T\left(a_{3} Z+a_{4} X Z+a_{5} X^{2}\right. \\
& \left.+a_{6} Y^{2} Z+a_{7} Z^{3} / 3\right)+h(X, Y),
\end{aligned}
$$

and from the $z$ component of the curl, using formulae relating the coefficients of the model $\left(a_{2}+b_{1}+2 c_{5}=0, a_{4}+b_{2}+c_{6}=0\right.$, $\left.2 a_{5}+b_{3}+c_{7}=0\right)$ :

$\partial h / \partial x-\partial g / \partial y=c_{1}+c_{2} X+c_{3} X^{2}+c_{4} Y^{2}$.

Putting $h=c_{1} X+c_{2} X^{2} / 2+c_{3} X^{3} / 3, g=-c_{4} Y^{3} / 3$ and suppressing the tilt-angle term, one obtains:

$$
\begin{aligned}
& A_{x}=b_{1} Y Z^{2} / 2-c_{4} Y^{3} / 3, \\
& A_{y}=-a_{1} Z^{2} / 2-a_{2} X Z^{2} / 2+c_{1} X+c_{2} X^{2} / 2+c_{3} X^{3} / 3, \quad A_{z}=0 .
\end{aligned}
$$

In all previous formulae concerning the external magnetic field, $X, Y$, and $Z$ are expressed in units of ten $\mathrm{R}_{\mathrm{E}}$. The field satisfies Faraday's law and quasi-neutrality condition.

\section{Appendix B}

The expression for the magnetic moment as a function of time with explicit dependence on field characteristics and on the initial characteristics of the ion will be derived. The equations of motion in the cyclotron plane are:

$v_{1}^{\prime}-b(t) v_{2}=e_{1}(t)$

$v_{2}^{\prime}+b(t) v_{1}=e_{2}(t)$

where $e_{1,2}(t)=q / m E_{1,2}(t)$ : the electric field components, $b(t)=q / m B(t)$. Functions $\sin (g(t)), \cos (g(t)), g(t)=\int b(t) d t$ comprise the fundamental set of solutions of homogeneous equations. The solutions of inhomogeneous equations can be found by making the constants time dependent:

$$
\begin{aligned}
& v_{1}=C(t) \cos g(t)+D(t) \sin g(t), \\
& v_{2}=-C(t) \sin g(t)+D(t) \cos g(t) .
\end{aligned}
$$


Solving the equations for $C(t), D(t)$ we obtain:

$C(t)=\int e_{1}(t) \cos g(t) d t-\int e_{2}(t) \sin g(t) d t+C_{0}$,

$D(t)=\int e_{1}(t) \sin g(t) d t-\int e_{2}(t) \cos g(t) d t+D_{0}$.

After changing the varible: $x=g(t), t=g^{-1}(x)$, the integrands transform into $e_{1} / b(x), e_{2} / b(x)$ factorized by $\sin (x)$ or $\cos (x)$. For a large class of functions able to approximate $e_{1} / b$ and $e_{2} / b$ as function of $x$, we have the property:

$\int f(x) \cos (x) d x=f_{s}(x) \sin (x)+f_{c}(x) \cos (x)$,

$\int f(x) \sin (x) d x=f_{c}(x) \sin (x)-f_{s}(x) \cos (x)$,

where $f_{s}, f_{c}$ are derivable if given the form of $f(x)$. After performing the algebra one obtains the expected expression for the magnetic moment:

$$
\begin{aligned}
2 B \mu / m= & \left|\boldsymbol{v}_{\perp}-\boldsymbol{V}_{D}\right|^{2} \\
= & C_{0}^{2}+D_{0}^{2}+\left[V_{D 1}-\left(f_{c 1}+f_{s 2}\right)\right]^{2}+\left[V_{D 2}-\left(f_{c 2}-f_{s 1}\right)\right]^{2} \\
& +2\left[\left(V_{D 2}-\left(f_{c 2}-f_{s 1}\right)\right) C_{0}\right. \\
& \left.-\left(V_{D 1}-\left(f_{c 1}+f_{s 2}\right)\right) D_{0}\right] \sin (g(t)) \\
& +2\left[-\left(V_{D 1}-\left(f_{c 1}+f_{s 2}\right)\right) C_{0}\right. \\
& \left.-\left(V_{D 2}-\left(f_{c 2}-f_{s 1}\right)\right) D_{0}\right] \cos (g(t)) .
\end{aligned}
$$

$V_{D 1,2}$ stand for the drift velocity components in the guiding center frame. The objective of further assumptions is to have a clear picture of the magnetic-moment changes during the motion in the timedependent magnetic and electric field. Let $e_{1}=e_{2}$ and $\left|V_{D 1}\right|=$ $\left|V_{D 2}\right|=V_{D}$. Then $V_{D}=\left[V_{D},-V_{D}\right], f_{s 1}=f_{s 2}=f_{s}, f_{c 1}=f_{c 2}=f_{c}$. If $\boldsymbol{v}_{\perp 0}=\left[v_{0} \cos (\varphi), v_{0} \sin (\varphi)\right]$ then $C_{0}=v_{0} \cos (\varphi)=C_{0}\left(v_{0}, \varphi\right), D_{0}=$ $v_{0} \sin (\varphi)=D_{0}\left(v_{0}, \varphi\right)$.

So

$$
\begin{aligned}
2 B \mu / m= & v_{\perp 0}^{2}+2 V_{D}^{2}-4 V_{D} f_{s}+2\left(f_{c}^{2}+f_{s}^{2}\right) \\
& -2 \sqrt{ } 2 v_{0} \sin (g(t))\left[\left(V_{D}-f_{s}\right) \sin (\pi / 4+\varphi)\right. \\
& \left.+f_{c} \sin (\pi / 4-\varphi)\right]-2 \sqrt{ } 2 v_{0} \cos (g(t)) \\
& \times\left[\left(V_{D}+f_{s}\right) \sin (\pi / 4+\varphi)-f_{c} \sin (\pi / 4+\varphi)\right] .
\end{aligned}
$$

It follows from Eq. B4 that the functions $f_{s}, f_{c}$ satisfy $f_{s}^{\prime \prime}+f_{s}=f$, $f_{c}=f_{s}^{\prime}$. If $f(x)=A \delta\left(x-x^{*}\right)$, then $f_{s}=A H\left(x-x^{*}\right) \sin \left(x-x^{*}\right)$, and $f_{c}=A H\left(x-x^{*}\right) \cos \left(x-x^{*}\right)$, where $\delta\left(x-x^{*}\right)$ is the delta-function and $H\left(x-x^{*}\right)$ is the Heavyside function; the number $A=\int_{-\infty}^{\infty} f(x) d x$. Including in the assumptions with Eq. B6 one obtains:

$$
\begin{aligned}
2 B \mu / m= & v_{\perp 0}^{2}+2 V_{D}^{2}+2 A^{2} H^{2}\left(x-x^{*}\right) \\
& +v_{0} A H\left(x-x^{*}\right) s_{0}(\varphi)-4 v_{0} V_{D} s_{1}(\varphi) \sin (\pi / 4+x) \\
& -4 V_{D} A H\left(x-x^{*}\right) \sin \left(x-x^{*}\right) \\
& +v_{0} A H\left(x-x^{*}\right) s_{2}\left(\varphi, x^{*}\right) \sin \left(2 x+s_{3}\left(\varphi, x^{*}\right)\right) .
\end{aligned}
$$

$$
\begin{aligned}
& s_{1}=\sin (\pi / 4+\varphi), \\
& s_{0}=2 s_{1} \cos \left(x^{*}+\pi / 4\right)+\sqrt{ } 2 \sin \left(x^{*}+C\right), \\
& \tan (C)=-\sin (\pi / 4+\varphi) / \sin (\pi / 4-\varphi),
\end{aligned}
$$

$$
\begin{aligned}
s_{2}^{2}=2+ & 4 s_{1}^{2}-4 \sqrt{ } 2 s_{1} \sin (\pi / 4+C), \\
\tan \left(s_{3}\right)= & \left(\sqrt{ } 2 \sin \left(-x^{*}+C\right)-2 s_{1} \cos \left(-x^{*}-\pi / 4\right)\right) / \\
& \left(\sqrt{ } 2 \cos \left(-x^{*}+C\right)+2 s_{1} \sin \left(-x^{*}-\pi / 4\right)\right)
\end{aligned}
$$

and $x^{*}=g\left(t=t^{*}\right)$, the point where the delta function is localized

\section{References}

Aggson, T. L., J. P. Heppner, and N. C. Maynard, Observations of large magnetospheric electric fields during the onset phase of a substorm, J. Geophys. Res., 88, 3981-3990, 1983.

Baker, D. N., E. W. Hones, Jr., D. T. Young, and J. Birn, The possible role of ionospheric oxygen in the initiation and development of plasma instabilities, Geophys. Res. Lett., 9, 1337-1340, 1982.

Chappell, C. R., T. E. Moore, and J. H. Wait Jr., The ionosphere as a fully adequate source of plasma for the Earth's magnetosphere, J. Geophys. Res., 92, 5896-5910, 1987.

Chen, F. F., Introduction to Plasma Physics and Controlled Fusion, vol. 1. Plasma Physics, Plenum Press, New York, 1984.

Daglis, I. A., E. T. Sarris, and G. Kremser, Indications for ionospheric participation in the substorm process from AMPTE/CCE observations, Geophys. Res. Lett., 17, 57-60, 1990.

Daglis, I. A., I. T. Sarris, and B. Wilken, AMPTE/CCE CHEM observations of the ion population at geosynchronous altitudes, Ann. Geophysicae, 11, 685-696, 1993.

Daglis, I. A., S. Livi, E. T. Sarris, and B. Wilken, Energy density of ionospheric and solar wind origin ions in the near-Earth magnetotail during substorms, J. Geophys. Res., 99, 5691-5703, 1994.

Delcourt, D. C., J. A. Sauvaud, and A. Pedersen, Dynamics of single-particle orbits during substorm expansion phase, J. Geophys. Res., 95, 20853-20865, 1990.

Ding C., T. W. Hill, and F. R. Toffoletto, Magnetic mapping and Birkeland currents in the Toffoletto-Hill and Tsyganenko magnetosphere models, J. Geophys. Res., 99, 17343-17350, 1994.

Fennell, J. F., D. R. Croley, Jr., and S. M. Kaye, Low-energy ion pitch angle distributions in the outer magnetosphere: ion zipper distribution, J. Geophys. Res., 86, 3375-3382, 1981.

Ganguli, S. B., H. G. Mitchell, and P. J. Palmadesso, Auroral plasma transport processes in the presence of $\mathrm{kV}$ potential structures, $J$. Geophys. Res., 99, 5761-5770, 1994.

Heikkila, W. J., and R. J. Pellinen, Localized induced electric field within the magnetotail, J. Geophys. Res., 82, 1610-1614, 1977.

Hultqvist, B., On the acceleration of electrons and positive ions in the same direction along magnetic field lines by parallel electric fields, J. Geophys. Res., 93, 9777-9784, 1988.

Jacquey, C., and J. A. Sauvaud, Magnetosphere-ionosphere response to an enhanced energetic coupling with the solar wind, J. Geophys. Res., 99, 11331-11339, 1994.

Kaye, S. M., E. G. Shelley, R. D. Sharp, and R. G. Johnson, Ion composition of zipper events, J. Geophys. Res., 86, 3383-3388, 1981.

Kistler, L. M., F. M. Ipavich, D. C. Hamilton, G. Gloeckler, B. Wilken, G. Kremser, and W. Stüdemann, Energy spectra of the major ion species in the ring current during geomagnetic storms, J. Geophys. Res., 94, 3579-3599, 1989.

Lu, G., P. H. Reiff, T. E. Moore, and R. A. Heelis, Upflowing ionospheric ions in the auroral region, J. Geophys. Res., 97, 16855-16863, 1992.

Lui, A. T. Y., R. E. Lopez, B. J. Anderson, K. Takahashi, L. J. Lanetti, R. W. McEntire, T. A. Potemra, D. M. Klumpar, E. M. Greene, and R. Strangeway, Current disruptions in the near-Earth neutral sheet region, J. Geophys. Res., 97, 1461-1480, 1992.

Lundqvist, P.-A., and G. T. Marklund, A statistical study of highaltitude electric fields measured on the Viking satellite, J. Geophys. Res., 95, 5867-5876, 1990.

Mauk, B. H., Quantitative modeling of the "convection surge" mechanism of ion acceleration, J. Geophys. Res., 91, 13423-13431, 1986. 
Mauk, B. H., Generation of macroscopic magnetic-field-aligned electric fields by the convection surge ion acceleration mechanism, J. Geophys. Res., 94, 8911-8920, 1989.

Mead, G. D., and D. H. Fairfield, A quantitative magnetospheric model derived from spacecraft magnetometer data. J. Geophys. Res., 80, 523-534, 1975.

Reiff, P. H., H. L. Collin, J. D. Craven, J. L. Burch, J. D. Winningham, E. G. Shelley, L. A. Frank, and M. A. Friedman, Determination of auroral electrostatic potentials using high- and low-latitude particle distributions, J. Geophys. Res., 93, 7441-7465, 1988.
Stern, D. P., One-dimensional models of quasi-neutral parallel electric fields, J. Geophys. Res., 86, 5839-5860, 1981.

Yau, A. W., B. A. Whalen, W. K. Peterson, and E. G. Shelley, Distribution of upflowing ionospheric ions in the high-altitude polar cap and auroral ionosphere, J. Geophys. Res., 89, 5507-5522, 1984

Yau, A. W., E. G. Shelley, W. K. Peterson, and L. Lenchyshyn, Energetic auroral and polar ion outflow at DE 1 altitudes: magnitude, composition, magnetic activity dependence, and long-term variations, J. Geophys. Res., 90, 8417-8432, 1985. 